\title{
Peertechz
}

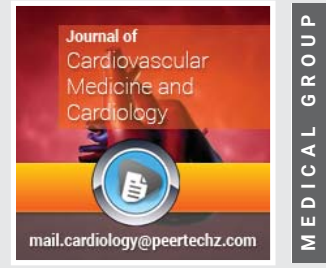

\section{A case of anomalous aortic origin of coronary arteries from the opposite sinus}

\author{
Alessandra Tanzilli, Simone Griffo, Alessandra De Luca \\ Vincenzo Capasso, Vincenzo Rossi, Marco Di Palma, \\ Simone Calcagno*, Francesco Panno, Riccardo Di Pietro, \\ Pino Aisle and Francesco Versaci
}

Division of Cardiology, S. Maria Goretti Hospital, Via Canova snc, 04100, Latina, Italy
Received: 30 July, 2020

Accepted: 05 August, 2020

Published: 06 August, 2020

*Corresponding author: Simone Calcagno, MD, Division of Cardiology, S. Maria Goretti Hospital, Via Canova snc, 04100, Latina, Italy, Tel: +3907736556217 Fax: +3907736556218;

E-mail: calcagnosimone@libero.it

ORCID: https://orcid.org/0000-0003-0129-471X

https://www.peertechz.com

Check for updates

\section{Abstract}

Anomalous Aortic Origin of Coronary Arteries (AAOCA) is a congenital heart defect in which one coronary artery arises from the opposite sinus of Valsalva. AAOCA is observed in approximately $0.3-1.3 \%$ of patients undergoing diagnostic Invasive Coronary Angiography (ICA). Among AAOCA, anomalous origin of the left coronary artery from the right sinus of Valsalva is a relatively uncommon finding, with a reported prevalence of $0.02 \%-0.05 \%$ on angiographic studies. This AAOCA is the second most common cause of death in young athletes and usually occurs during exertion. Several imaging modalities have been proposed for AAOCA identification and evaluation. Multidetector Computed Tomography (MDCT) may not only visualize AAOCA but better define, as compared with ICA, the origin and course of the anomalous vessel. We report a case of a 19 years old young athlete with anomalous origin of the Left Main Coronary Artery (LMCA) from the right sinus of Valsalva that was detected by MDCT using prospective ECG gating and low-radiation exposure.

\section{Introduction}

Anomalous origin of the left coronary artery from the right sinus is a relatively uncommon finding, with a reported prevalence of $0.02 \%-0.05 \%$ on angiographic studies [1]. Nevertheless, it is a leading cause of Sudden Cardiac Death (SCD) in young athletes and its clinic manifestations are strictly related to strenuous exertion. It is thought that the main mechanism of SCD relies on external compression of the abnormal coronary artery, coursing between the great arteries, as they expand during vigorous exercise, leading to myocardial ischemia. Usually, death is unexplained, but in the majority of the cases it could present with exertional angina or Electrocardiographic (ECG) signs of stress induced myocardial ischemia even totally asymptomatic. It is widely known that the prognosis of this coronary anomaly changes-according to anatomical variant. Consequently, an early recognition and a detailed definition of critical anatomical variant and its functional significance is of a paramount importance, Multislices Coronary Computed Tomography (MSCCT) is a powerful diagnostic tool that can provide optimal visualization and characterization of the vessel coronary arteries origin and their anatomical course subtypes. We report the case of a young athlete.

\section{Case presentation}

A 19-year-old athlete Caucasian male was referred to our department for assessment of coronary artery anatomy by Multislices Coronary Computed Tomography (MSCCT) due to ischemia induced exercise stress test performed during sports pre-participation screening. He was asymptomatic for dyspnea, chest pain or other ischemic myocardial equivalent. Physical examination did not reveal any abnormality. The basal ECG showed regular sinus rhythm without signs of ischemia or prior myocardial infarction.

Transthoracic echocardiography demonstrated a preserved LV ejection fraction (61\%) without regional wall motion abnormalities. There was mild MR from a thick, restricted posterior leaflet. A 24 hours record ECG showed ST-T segment no-specific changes associated with the increase of heart rate. 
A maximal electrocardiographic exercise stress test performed according to Bruce's criteria showed significant ST segment downsloping in II, III and aVF peripheral leads and a right bundle branch block (RBBB) appearance. The patient was asymptomatic during the test.

MSCCT was conducted with a new ultrafast-low-dose protocol (Revolution CT, General Electric) with volumetric acquisition technique and retrospective cardiac gating [2], after administration of $80 \mathrm{ml}$ of a tri-iodinated non-ionic monomeric contrast medium e.v. (IOMERON ${ }^{\circledR} 400 \mathrm{mg} / \mathrm{ml}$, Bracco Imaging S.p.A). The scans obtained were reconstructed at different stages of the cardiac cycle and analyzed with multiplanar algorithms and volumetric rendering.

Volume rendering reconstruction showed the left main and right coronary artery (RCA) arising separately from the right sinus of Valsalva (Figure 1). The left main coursed between the ascending aorta and pulmonary trunk (Figure 2) and gave off the left anterior descending (LAD) and left circumflex (LCX) coronary arteries in their normal position.

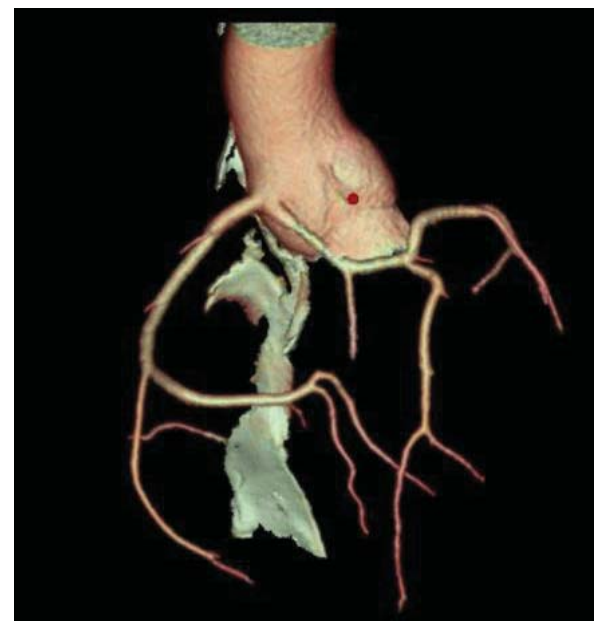

Figure 1: Volume rendering reconstruction showed the left main and right coronary artery (RCA) arising separately from the right sinus of Valsalva.

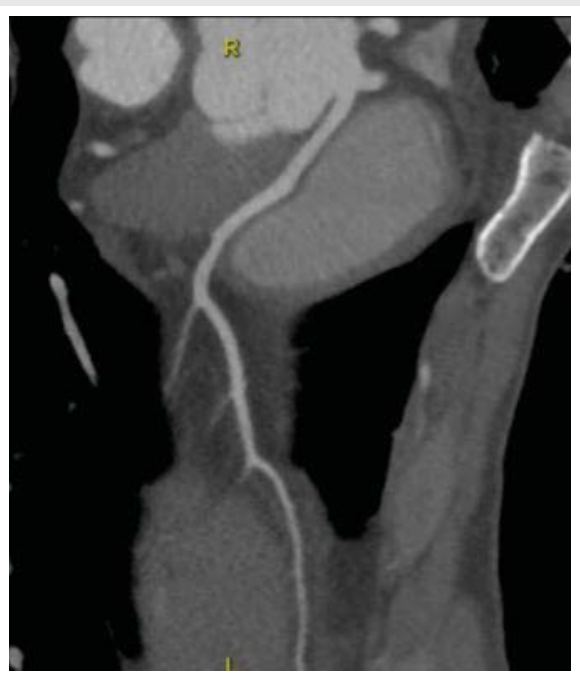

Figure 2: The left main coursed between the ascending aorta and pulmonary trunk and gave off the left anterior descending (LAD) and left circumflex (LCX) coronary arteries in their normal position.

\section{Discussion}

Currently, coronary Computed Tomography (CTA) and MRI are the only Class I-indicated tests used to image Anomalous Aortic Origin of a Coronary Artery (AAOCA) [3]. The recent advances in MSCCT Multi-Detector Row Computed Tomography (MDCT) technology have allowed this technique to become the method of choice for detecting coronary artery anomalies. In particular, rapid scan times, high spatial resolution and dose reduction strategies have improved patient safety $[4,5]$. Noteworthy, our method routinely permits very low radiation exposures $(<2 \mathrm{mSv})$, low contrast volumes, and rapid imaging by free breathing without sedation. MDCT is the best non-invasive imaging technique in terms of sensibility, specificity, positive and negative predictive value as regard this group of malformations [6]. Non-contrast Self-navigated Free-breathing MR Angiography (SN3D-MRA) studies provide considerable diagnostic accuracy regarding the detection of anomalies, high origin, and inter-arterial course of the coronary arteries. However, contrast enhanced CTA remains the superior imaging technique currently in terms of diagnostic confidence and visualization of more distal coronary artery branches and characterization of subtle anatomical detail such as intramural coronary course [7].

Based on the functional relevance of each abnormality, coronary artery anomalies can be classified as anomalies with obligatory ischemia, without ischemia or with exertional ischemia.

Despite these differences in prevalence rates between different variant of abnormality the AAOCA described in our case, is the second most common cause of death in young athletes and usually occurs during exertion and is associated with the risk of Sudden Cardiac Death (SCD ) and as such should be carefully considered in symptomatic patients [8]. Clinical symptoms of AAOCA are similars to symptoms of atherosclerotic coronary artery disease, especially when the anomalous artery has an interarterial course. Of note, when a subject undergoes strenuous activity, the increased cardiac output results in a greater expansion of the aorta and pulmonary artery. In the presence of inter-arterial course of the right-sided AAOCA, as seen in our patient, it is possible that the enlarged vessels mechanically compress the LCA, effectively acting as an exercise-dependent form of stenosis. In addition, the assessment of different subtypes of coronary artery anomalies provides better risk stratification of the patients. The proximal course of the anomalous coronary artery seems a major determinant of the risk of myocardial infarction or sudden death. In particular, the high-risk course between the aorta and the pulmonary trunk must be discriminated from rather innocent low-risk septal and anterior infundibular courses $[9,10]$.

Although this report outlines a rare case of asymptomatic right-sided AAOCA, it is generally considered to be more benign than other coronary artery anomalies, such as a leftsided AAOCA. The difference in mortality between the 2 types of AAOCA has been described in past autopsy reports, with a mortality rate of $57 \%$ for left-sided AAOCA and $25 \%$ for right- 
sided AAOCA. As widely described, diagnosis of AAOCA is usually incidental as most patients are asymptomatic. In those who do present with symptoms, the most common ones are exertional syncope, chest pain, or palpitations. Symptomatic AAOCA patients have 3 treatment options: medical treatment, coronary angioplasty with stenting, and surgical repair, which includes bypass surgery, reimplantation of the anomalous artery, unroofing of intramural segments of the anomalous artery, or osteoplasty. In conclusion, although AAOCA is fairly rare, the potential risk of SCD and other adverse complications make accurate diagnosis and treatment of this condition crucial to maximizing patient outcomes.

\section{References}

1. Flessas D, Mamarelis I, Maniatis V, Souretis G, Laschos N, et al. (2013) An unusual pattern of three major components of the cardiovascular system: Multimodality imaging and review of the literature. J Cardiothorac Surg 8: 61 . Link: https://bit.ly/30uyod1

2. Gaudio C, Petriello G, Pelliccia F, Tanzilli A, Bandiera A, et al. (2018) A nove ultrafast-low-dose computed tomography protocol allows concomitant coronary artery evaluation and lung cancer screening. BMC Cardiovasc Disord 18: 90. Link: https://bit.ly/2C01wzj

3. Warnes C, Williams RG, Bashore TM, Child JS, Connolly HM, et al. (2008) ACC/AHA 2008 guidelines for the management of adults with congenital heart disease: a report of the American College of Cardiology/ American Heart Association Task Force on Practice Guidelines. J Am Coll Cardiol 52 :e143-e263. Link: https://bit.ly/39XPh2B

4. Halliburton SS, Abbara S, Chen MY, Gentry R, Mahesh M, et al. (2011)
SCCT guidelines on radiation dose and dose-optimization strategies in cardiovascular CT. J Cardiovasc Comput Tomogr 5: 198-224. Link: https://bit.ly/2PmTuUe

5. Chinnaiyan KM, Boura JA, DePetris A, Gentry R, Abidov A, et al. (2013) Progressive radiation dose reduction from coronary computed tomography angiography in a statewide collaborative quality improvement program: Results from the advanced cardiovascular imaging consortium. Circ Cardiovasc Imaging 6: 646-654. Link: https://bit.ly/3kdzSjA

6. Yin X, Wang J, Zheng W, Ma J, Hao P, Chen Y (2016) Diagnostic performance of coronary computed tomography angiography versus exercise electrocardiography for coronary artery disease: A systematic review and meta-analysis. J Thorac Dis 8: 1688-1696. Link: https://bit.ly/2ETNctd

7. Albrecht MH, Varga-Szemes A, Joseph Schoepf U, Nance JW, De Cecco CN, et al. (2019) Diagnostic Accuracy of Noncontrast Self-navigated Free-breathing MR Angiography versus CT Angiography: A Prospective Study in Pediatric Patients with Suspected Anomalous Coronary Arteries. Acad Radiol 26: 13091317. Link: https://bit.ly/3fuzAkN

8. Cheezum MK, Liberthson RR, Shah NR, Villines TC, O'Gara PT, et al. (2017) Anomalous Aortic Origin of a Coronary Artery from the Inappropriate Sinus of Valsalva. Journal of the American College of Cardiology 69. Link: https://bit.ly/31grbwa

9. Barth CW, Roberts WC (1986) Left main coronary artery originating from the right sinus of valsalva and coursing between the aorta and pulmonary trunk. $J$ Am Coll Cardiol 7: 366-373. Link: https://bit.ly/31m5hrl

10. Kragel AH, Roberts WC (1988) Anomalous origin of either the right or left main coronary artery from the aorta with subsequent coursing between aorta and pulmonary trunk: Analysis of 32 necropsy cases. Am J Cardiol 62: 771-777. Link: https://bit.ly/33uWPJ4
Discover a bigger Impact and Visibility of your article publication with Peertechz Publications

Copyright: (c) 2020 Tanzilli A, et al. This is an open-access article distributed under the terms of the Creative Commons Attribution License, which permits unrestricted use, distribution, and reproduction in any medium, provided the original author and source are credited.

Citation: Tanzilli A, Griffo S, Luca Vincenzo Capasso AD, Rossi V, Calcagno S, et al. (2020) A case of anomalous aortic origin of coronary arteries from the opposite sinus. J Cardiovasc Med Cardiol 7(3): 232-234.DOI: https://dx.doi.org/10.17352/2455-2976.000144 